\title{
Acontecimientos vitales y adicción: una revisión de la bibliografía
}

\author{
Hussein El-Shikh, MSc, MD, Assistant \\ professor in psychiatry* \\ Ehsan Fahmy, MSc, MD, Professor of \\ Psychiatry* \\ Victor Samy Michael, MSc, MD, Professor \\ of Psychiatry* \\ Hamdy Fouad A Moselhy, MSc, \\ MRCPsych, consultant Psychiatrist * \\ * Benha Faculty of Medicine, Zagazig \\ University \\ ** Sandwell Mental Health NHS Trust, Anchor \\ Project \\ UNITED KINGDOM
}

\begin{abstract}
RESUMEN - La idea de que los acontecimientos vitales estresantes pueden contribuir al inicio y desarrollo de un trastorno por abuso de sustancias ha ganado aceptación, particularmente en la comunidad clínica. Se revisan aquí las investigaciones sobre acontecimientos vitales y su relación con los trastornos adictivos. Los acontecimientos adversos de la vida diaria se dividirán en acontecimientos predisponentes o de vulnerabilidad, precipitantes o desencadenantes, y factores mediadores. Se resumen los hallazgos de los estudios y se argumenta su relevancia para la metodología.
\end{abstract}

\section{Introducción}

Se ha demostrado que el estrés vital en los adultos puede provocar problemas tanto en la salud física como en la salud psicológica. Existe un considerable volumen de datos sobre la posible conexión entre los acontecimientos que ocurren durante el curso de la vida y el inicio de la enfermedad
(O’Doherty y Davies 1987). El trabajo de Brown y Harris (1978) es uno de los ejemplos más destacados en el área de investigación sobre depresión. La investigación sobre los acontecimientos vitales y su relación con la adicción es un área mucho menos explorada. El complejo concepto de acontecimientos vitales implica no sólo que las enfermedades psiquiátricas son iniciadas o perpetuadas por interacciones entre el 
entorno y el individuo, sino que los acontecimientos vitales concretos modifican esa interacción. En algunos casos la vulnerabilidad llega a ser más aguda, en otros menos, y lo mismo ocurre en los acontecimientos externos.

Investigaciones anteriores han documentado una mayor frecuencia de acontecimientos vitales para los consumidores de droga previos al comienzo del consumo, comparados con grupos control y de pacientes psiquiátricos emparejados. Hay también datos de una mayor incidencia de acontecimientos vitales tras el comienzo del consumo, cuando se compara gente que se inyecta drogas con un grupo control (O’Doherty y Davies 1987).

En este artículo revisaremos los diferentes aspectos de los acontecimientos vitales y su relación con la dependencia a drogas.

\section{Acontecimientos vitales y adicción}

Actualmente se acepta ampliamente que hay dos variables psicosociales entre los acontecimientos vitales, que juegan un papel significativo en el desarrollo de las adicciones: las experiencias en la niñez (Kubicka y Kozeny 1988, Bernardi et al. 1989) y, posteriormente, traumas en adultos (Rousanville et al. 1982, Cooke y Hole 1983). Sin embargo, los mecanismos que median el efecto de estas variables son referidos como mediadores. Estos factores de riesgo mediados han sido estudiados en los trastornos depresivos (Farmer et al. 2002), donde el neuroticismo y la extraversión correlacionaron con el estado de ánimo y los acontecimientos vitales.

Para argumentar la relación entre el exceso de acontecimientos vitales, otros factores mediadores y la adicción, condensaremos los estudios en tres grupos: a) acontecimientos negativos en la niñez como situaciones de vulnerabilidad, b) acontecimientos negativos en la edad adulta como desencadenantes, y c) factores de riesgo mediados.

\section{Acontecimientos negativos en la niñez y situaciones de vulnerabilidad}

Los acontecimientos vitales juegan un papel establecido en la morbilidad psiquiátrica en la niñez, incluídos niños consumidores de sustancias. Por ejemplo, se encontró que las tasas más altas de duelo (Stoker y Swadi 1990) de enfermedad grave ("major") (Brook et al. 1989) y de abuso sexual en la niñez o en la adolescencia (Hernandez 1992) eran más prevalentes entre los adolescentes consumidores de drogas que entre los no consumidores.

Las características del niño, las de los padres y las de la relación única que existe entre ellos afectan el comportamiento futuro del niño. Un gran número de autores han investigado las conductas observables que expresan los estilos parentales entre el padre y la descendencia (O’Kearney 1996). El impacto de los estilos de paternidad en la niñez representa un campo muy amplio de investigación, incluyendo el área de la adicción, y ha producido un gran número de estudios.

Se ha documentado que las experiencias disciplinarias en la niñez tienen un impacto en los trastornos afectivos y adictivos (Holmes y Robins 1987). Sin embargo, la información proporcionada por Holmes y Robins sobre su procedimiento de reclutamiento es limitada. 
Otro estudio sobre las adversidades en la niñez investigó la relación temporal que tiene la adicción al opio con la psicopatología y la criminalidad. Rousanville et al. (1982) entrevistaron a un amplio grupo de sujetos $(n=363)$ con adicción al opio, sobre su niñez, sobre el comienzo de su comportamiento delictivo regular y sobre el inicio ilícito de drogas. Encontraron tres subgrupos de consumidores de opio: un grupo inicial de niños con algún trauma; un grupo de delincuentes a edad temprana; y un grupo expuesto tanto a drogas como a la presión de los compañeros de grupo. Hay evidencia que confirma la observación probada de que el abandono del colegio puede ser un factor de riesgo significativo para el consumo posterior de drogas (Robins 1980). También, hay consistencia significativa en la evidencia que indica agresión y comportamiento antisocial en la niñez temprana como factor de riesgo principal para el consumo de drogas (Hawkins et al. 1992).

La morbilidad psiquiátrica general en la niñez, independientemente del tipo de patología, se ha identificado como un factor de riesgo (Lopes et al. 1996). Los factores más comúnmente investigados son los trastornos de conducta, el trastorno por déficit de atención e hiperactividad (ADHD) y los trastornos afectivos (Horner y Scheibe 1997). Los estudios anteriormente citados han subrayado la importancia de realizar mediciones de las adversidades de la niñez cuando se estudia el origen de las adicciones.

Hay evidencia de que el consumo de sustancias de los padres y su comportamiento juegan un papel importante. Se sabe muy poco sobre el consumo de drogas de los padres. La información disponible sugiere que las actitudes parentales permisivas hacia el consumo de drogas y el consumo de drogas por parte de los padres incrementa el consumo de drogas de los adolescentes (Kandel
1982). Varios estudios documentaron un incremento en el consumo de alcohol entre los adolescentes descendientes de padres alcohólicos (Swadi 1999). Sin embargo, otros no encontraron tal correlación (Roosa et al. 1988, Johnson et al. 1989). No obstante, se documentaron mayores tasas de abuso de drogas entre los adolescentes con padres alcohólicos (Johnson et al. 1989). El consenso es que los niños de padres que abusan de sustancias presentan un riesgo mayor de desarrollar trastornos relacionados con el abuso de sustancias, incluyendo los relacionados con el abuso de alcohol.

Las dinámicas familiares y los estilos parentales, tales como el divorcio de los padres, la discordia entre los padres, la ruptura familiar, la comunicación negativa, la respuesta afectiva, el desarrollo de los roles, la disciplina inconsistente de los padres, y la violencia familiar, se han identificado como situaciones estresantes importantes en la vida de los consumidores de drogas (Stoker y Swadi 1990). Las familias de los niños que consumen drogas estaban caracterizadas por padres distantes y no implicados y madres desbordadas (Kaufman y Kaufman 1979).

Para los adictos a narcóticos, se han postulado tres grandes tipos de trastornos: provenir de "hogares rotos", sobreprotección de los padres, y sistemas familiares en los que hay algún tipo de refuerzo de las conductas adictivas por miembros familiares (Blechman 1982). Stimson (1973) documentó que cerca de la mitad de los adictos a la heroína habían experimentado una separación de al menos un año de sus padres antes de los 16 años, mientras que Rounsaville et al. (1982) encontraron que un tercio de su muestra había experimentado acontecimientos "perjudiciales" en la niñez, en particular violencia, hospitalización y separación de los padres. Tenant y Bernardi (1988) documen- 
taron que la pérdida de los padres en la niñez es más común en los adictos a narcóticos y en los alcohólicos que en los controles. El tipo de separación normalmente implicado es el resultado del conflicto familiar o marital. En otro informe, los autores compararon a los alcohólicos y a los adictos a heroína con un grupo control normal y encontraron que la sobreprotección maternal y paternal era más frecuente en los adictos a narcóticos (Bernardi et al. 1989).

Parker et al. (1979) utilizando el Parental Bonding Instrument (PBI) encontraron que los clientes que referían niveles bajos de atención y niveles altos de sobreprotección durante la niñez habían desarrollado con más probabilidad problemas psicológicos en la edad adulta.

El PBI se ha utilizado también específicamente para estudiar la calidad de la paternidad en consumidores de alcohol y drogas (Bernardi et al. 1989). Encontraron niveles altos de sobreprotección maternal y paternal en los consumidores de drogas, comparado con un grupo control. El grupo de alcohólicos mostró niveles altos de sobreprotección maternal, pero no paternal. No se encontraron diferencias significativas entre el grupo de alcohólicos y el de adictos a drogas. No se hallaron diferencias ni en el cuidado paterno ni en el materno, entre los tres grupos.

Cualquier investigación basada en el PBI puede ser criticada en el terreno de la hipótesis en "búsqueda de explicación", por la que una persona con un trastorno intenta explicar su origen mediante la búsqueda de un acontecimiento causal. Desafortunadamente, es más probable que éste sea el caso de las personas con dificultades mentales, más que en sujetos sanos. Esto podría, por tanto, influir en las respuestas de cualquier grupo clínico cuando se enfrenta con preguntas retrospectivas.
Tennant y Bernardi (1988) probaron la hipótesis del "hogar roto", comparando las experiencias de separación en la niñez entre los tres grupos. Encontraron que ambos grupos clínicos tenían frecuencias y duraciones más altas de las pérdidas maternas y de la pérdida de ambos padres, comparado con los grupos control. No hubo diferencias significativas en las pérdidas paternas entre los tres grupos. El aumento en la separación maternal, en los grupos clínicos, se explicó por una mayor incidencia de conflictos maritales en estos grupos, mientras que la separación por fallecimiento no mostró variación entre los tres grupos.

Tanto Bernardi et al. (1989) como Tennant y Bernardi (1988) señalaron las dificultades con la madre durante la niñez como indicadores del abuso de sustancias posterior. Esto enfatiza la importancia de la madre como la figura principal en la niñez. Gorman y Brown (1992) discutieron la idea de que ciertas personas son más "predispuestas a acontecimientos" con respecto a algunos tipos específicos de eventos, y suscita la cuestión de hasta qué punto las predisposiciones de personalidad pueden influir la relación entre los acontecimientos vitales y las conductas adictivas.

\section{Acontecimientos vitales negativos en adultos como desencadenantes de trastornos}

La mayoría de estados depresivos y ansiosos parecen estar desencadenados por un acontecimiento vital grave unos meses antes del inicio (Brown 1981). Entonces, podría hipotetizarse que un trastorno más complejo como la adicción puede ser consecuencia de una adversidad más grave y, sólo cuando parece no haber disponible otra conducta alternativa, se desarrollaría la adicción. Un 
agotamiento constante de los recursos de una persona puede conducir al empleo de cualquier estrategia de afrontamiento disponible, incluso si es ilegal y/o se sabe que es dañino (Alexander y Hadaway 1982).

O’Doherty y Davies 1988, criticaron duramente la metodología retrospectiva e intentaron superar las limitaciones del recuerdo sugiriendo el uso de una entrevista recurrente. En su artículo de 1987 compararon tres grupos de consumidores de sustancias (alcohol, heroína y tabaco) con un grupo de sujetos controles, para investigar el origen de la adicción. O’Doherty y Davies concluyeron que los sujetos de los grupos de consumidores de drogas y de alcohol no experimentaron más acontecimientos vitales (independientemente de la adicción), pero sí que los acontecimientos se interpretaban de forma diferente a los que les pasaban al otro grupo. Sin embargo, su estudio se concentró en las razones de por qué la gente abusaba de las drogas una vez que había desarrollado la adicción, y no diferenciaron entre la etiología y el mantenimiento.

El embarazo en adolescentes parece ser un acontecimiento especialmente de riesgo. Los estudios mostraron una mayor incidencia de adolescentes embarazadas que abusaban de las drogas y que bebían alcohol (Cornelius et al. 1994). Sarvela y Ford (1992) señalaron que el riesgo era particularmente alto entre las adolescentes embarazadas con problemas de salud mental. En las mujeres, el acoso sexual, violación y otras experiencias traumáticas incrementaron el riesgo de consumo problemático de alcohol (Resnick et al. 1997).

También los individuos expuestos a acontecimientos traumáticos violentos pueden estar expuestos a mayor riesgo de abusar de drogas y de padecer síntomas depresivos (Kilpatrick et al. 1997). Clark et al. (2001) encontraron que los síntomas relacionados con el trastorno de estrés post-traumático (TEP) estaban asociados a un mayor consumo de drogas grave controlando por edad, depresión, y diagnóstico de TEP a lo largo de la vida. Otros estudios confirmaron estos datos (Kessler 1995, Sims y Sims 1998).

\section{Factores mediadores}

Los mecanismos que median los efectos de los acontecimientos vitales como factores de riesgo a menudo son referidos como "mediadores" (Willis et al. 1995). Dichos mecanismos han ganado atención en estudios recientes. Sin embargo, la relación entre acontecimientos vitales como factores de riesgo y sus mediadores puede ser de naturaleza muy compleja. Cloninger (1987) fue el primero que describió ciertas características de la personalidad de los individuos en riesgo de desarrollar un alcoholismo de inicio temprano. Posteriormente, Cloninger et al. (1988) apoyaron esta teoría en un estudio en 431 hombres suecos. A pesar de que la teoría de Cloninger se centraba en el consumo de alcohol, se ha probado empíricamente para otras sustancias (Willis et al. 1994).

Otra investigación documentó que las características de dificultad temperamental en la niñez temprana incrementaban la posibilidad de consumir tabaco, alcohol y marihuana en la adolescencia (Lerner y Vicari 1984). Blackson y Tarter (1994) estudiaron los factores de afiliación individuales, familiares y de iguales, que predisponen al inicio en el consumo de alcohol y drogas a edad temprana. Los atributos de personalidad en la niñez parecen ser factores de riesgo para el inicio temprano en el consumo de sustancias (Smith et al. 1982). 
Las influencias de compañeros parecen tener, según la mayor parte de las comunicaciones, el mayor impacto en la conducta adolescente de abuso de sustancias (Jenkins 1996). El consumo con compañeros se ha identificado universalmente como el factor que mejor predice el consumo de drogas actual (Swedi 1999). El consumo de drogas parece servir de herramienta social para algunos adolescentes. Parece proporcionar un ambiente social y un marco favorable para el consumo de drogas. Tal marco incrementa el riesgo de iniciación, así como el consumo continuado (Drupe et al. 1995, Fergusson et al. 1995).

Los estudios previos indicaron que las drogas se relacionan con la delincuencia y con otras conductas ilegales (Elliot et al. 1982). Esto se ha confirmado en estudios recientes (Brook et al. 1996). La carencia de hogar (sin techo, "homelessness") se ha implicado también como un factor mediador (Johnson et al. 1997). Sin embargo, debemos considerar el modelo bi-direccional, donde el consumo de sustancias puede ser un factor de riesgo para llegar a ser un "sin techo".

\section{Consideraciones metodológicas}

\section{Medidas y análisis retrospectivos}

La mayoría de estudios en el campo de los acontecimientos vitales y de la adicción han utilizado una recogida retrospectiva de datos (O’Doherty y Davies 1987). La objeción principal al análisis retrospectivo es: memoria deficitaria en el sujeto poco fiable (Todd 1966). Además, los individuos tratan de dar sentido a sus vidas. Esto puede conducir a que perciban los acontecimientos pasados como influyentes en su conducta posterior.

\section{Tamaño muestral}

Algunos estudios sobre acontecimientos vitales y consumo de drogas han empleado grupos altamente seleccionados, en los que sólo se contactó con pacientes de muestras clínicas, ignorando la amplia población de la comunidad desconocida en los servicios sanitarios (Tennant y Bernardi 1988, Bernardi et al. 1989).

\section{Grupos control}

La información retrospectiva sobre la frecuencia de acontecimientos vitales en un grupo de sujetos puede interpretarse con sentido solamente en comparación con información similar de grupos control apropiados. En algunos de los estudios revisados, el grupo control fue o bien un grupo de la población normal o bien otro grupo en tratamiento. Sin embargo, el sujeto debería emparejarse en base a variables sociales y otras variables relevantes.

\section{Comienzo/instauración del cuadro}

Una definición clara del comienzo es esencial en cualquier estudio sobre la etiología de los trastornos, incluyendo el abuso de sustancias. Hay algunos autores que han tratado la cuestión de la contaminación, que puede llevar a incluir acontecimientos resultantes del trastorno actual en el análisis de agentes causales (Gorman 1987). Uno de los enfoques en el campo de la adicción ha sido definir el comienzo como los primeros síntomas de adicción que continúan y even- 
tualmente se desarrollan hasta llegar a presentarse con una frecuencia con la que se puede hacer el diagnóstico de trastorno (Troop y Treasure 1997).

\section{Conclusión}

En los últimos años se han publicado numerosos estudios en el campo de los acontecimientos vitales y la adicción. Se han identificado gran número de acontecimientos vitales como capaces de incrementar la posibilidad de desarrollar un consumo de drogas. Sin embargo, no ha emergido un modelo claro de todo este trabajo. Por tanto, la investigación sobre los acontecimientos vitales necesita, cada vez más, concentrarse en examinar los procesos donde acontecimientos vitales específicos interaccionan con la vulnerabilidad biológica y psicosocial predisponente para producir dogodependencia.

\section{Bibliografía}

ALEXANDER, B.K., HADAWAY, P.F. Opiate addiction: the case for an adaptive orientation. Psychological Bulletin, 92, 367-381, 1982.

BERNARDI, E., JONES, M., TENNANT, C. Quality of parenting in alcoholics and narcotic addicts. The British Journal of Psychiatry, 154, 677-682, 1989.

BLACKSON, T., TARTER, R. Individual, family, and peer affiliation factors predisposing to early-age onset of alcohol and drug use. Alcohol Clinical and Experimental Research, 18, 813-821, 1994.

BLECHMAN, E. Conventional wisdom about familial contributions to substance abuse. American Journal of Drug and Alcohol Abuse, 9(1), 35-53, 1982.

BROOK, J., NOMURA, C., COHEN, P. Parental, perinatal, and early childhood risk factors and drug involve- ment in adolescence. Genetic Sociology of General Psychology Monographs, 115, 221-241, 1989.

BROOK, J., WHITEMAN, M., FINCH, S., COHEN, P. Young adult drug use and delinquency: Childhood antecedents and adolescent mediators. Journal of American Academy of Child and Adolescent Psychiatry, 35, 1584-1592, 1996.

BROWN, G.W. Life events, psychiatric disorder and physical illness. Journal of Psychosomatic Research, 25, 461-473, 1981

BROWN, G.W., HARRIS, T.O. Social origins of depression: A study of psychiatric disorder in women. Tavistock, London, 1978.

CLARK, H.W., MASSON, C.L., DELUCCHI, K.L., HALL, S.M., SEES, K.L. Violent traumatic events and drug abuse severity. Journal of substance Abuse Treatment, 20, 121-127, 2001.

CLONINGER, C. Neurogenetic adaptive mechanisms in alcoholism. Science, 236, 410-416, 1987.

CLONINGER, C., SIGVARDSSON, S., BOHMAN, M. Childhood personality predicts alcohol use in young adults. Alcoholism, 12, 494-505, 1988.

COOKE, D.J., HOLE, D.J. The aetiological importance of stressful life events. The British Journal of Psychiatry, 143, 397-400, 1983

CORNELIUS, M., RICHARDSON, G., DA, N., CORNELIUS, J., GEVA, D., TAYLOR, P. A comparison of parental drinking in two recent samples of adolescents and adults. Journal of Study in Alcohol, 55, 412-419, 1994.

DUPRE, D., MILLER, N., GOLD, M. Initiation and progression of alcohol, marijuana and cocaine use among adolescent abusers. American Journal of Addiction, 4, 4348, 1995 .

ELLIOTT, D., HUIZINGA, D., AGETON, S. Explaining delinquency and drug use. Behavioural Research Institute, Boulder Report n. ${ }^{\circ} 2,1982$.

FARMER, A., REDMAN, K., HARRIS, T., MAHMOOD, A., SADLER, S., PICKERING, A., McGUFFIN, P. Neuroticism, extraversion, life events and depression. The British Journal of Psychiatry, 181, 118-122, 2002.

FERGUSSON, D.M., HORWOOD, L.J., LYNSKY, M.T. The prevalence and risk factors associated with abusive or hazardous alcohol consumption in 16 year olds. Addiction, 90, 935-946, 1995.

GORMAN, D.M. Measuring onset of caseness in studies of stressful life events and alcohol abuse. British Journal of Addiction, 82, 1017-1020, 1987. 
GORMAN, D.M., BROWN, G.W. Recent development in life-event research and their relevance for the study of addictions. British Journal of Addiction, 87, 837-8849, 1992.

HAWKINS, J., CATALANO, R., MILLER, J. Risk and protective factors for alcohol and other drug problems in adolescence and early adulthood: implications for substance abuse prevention. Psychology Bulletin, 112, 64-105, 1992.

HERNANDEZ, J. Substance abuse among sexually abused adolescents and their families. Journal of Adolescent Health, 13, 658-662, 1992.

HOLMES, S.J., ROBINS, L.N. The influence of childhood disciplinary experience on the development of alcoholism and depression. Journal of Child Psychology and Psychiatry, 28, 399-415, 1987.

HORNER, B., SCHEIBE, K. Prevalence and implications of attention deficit hyperactivity disorder among adolescents in treatment for substance abuse. Journal of American Academy of Child and Adolescence Psychiatry, 36, 30-36, 1997.

JENKINS, J.E. The influence of peer affiliation and student activities on adolescent drug involvement. Adolescence, 31, 297-306, 1996.

JOHNSON, S., LEONARD, K., JACOB, T. Drinking, drinking styles and drug use in children of alcoholics, depressives and controls. Journal of Studies in Alcohol, 50, 427-431, 1989.

JOHNSON, T., FREELS, S., PARSON, J. Substance abuse and homelessness: social selection or social adaptation? Addiction, 92, 437-445, 1997.

KANDEL, D. Epidemiological and psychosocial perspectives on adolescent drug use. Journal of American Academy of Child Psychiatry, 21, 328-347, 1982.

KAUFMAN, E., KAUFMAN, P. Family therapy of drug and alcohol abuse. Gardener Press, New York, 1979.

KESSLER, R.C., SONNEGA, A., BROMET, E.J., HUGHES, M., NELSON, C.B., Posttraumatic stress disorder in the National Comorbidity Survey. Archives of General Psychiatry, 52, 1048-1060, 1995.

KILPATRICK, D.G., ACIERNO, R., RESNICK, H.S., SAUNDERS, B.E., BEST, C.L. A 2 year longitudinal analysis of the relationships between violent assault and substance use in women. Journal of Consulting and Clinical Psychology, 65, 834-847, 1997.

KUBICKA, L., KOZENY, J. Psychosocial variables related to quantity of alcohol consumed at a single session by young Czech males. British Journal of Addiction, 83, 1163-1168, 1988.
LERNER, J., VICARY, J. Difficult temperament and drug use: analysis from the New York Longitudinal study. Jornal of Drug education, 14, 1-7, 1984.

LOPES, C., LEWIS, G., MANN, A . Psychiatric and alcohol disorders as risk factors for drug abuse. A case-control study among adults in Rio de Janeiro, Brazil. Social Psychiatry and Psychiatric Epidemiology, 31, 355-363, 1996.

O'DOHERTY, F., DAVIES, J.B. Life events and addiction: a critical review. British Journal of Addiction, 82, 127137, 1987.

O'DOHERTY, F., DAVIES, J.B. Life events, stress and addiction. In: Fisher S and Reason $\mathrm{J}$ (eds) Handbook of life stress, cognition and health. John Wiley and Sons Ltd, London, 1988.

O'KEARNEY, R. Attachment disruption in anorexia nervousa and bulimia nervousa: A review of theory and empirical research. International Journal of Eating Disorders, 20, 115-127, 1996.

PARKER, G., TUPLING, H., BROWN, L.B. A parental bonding instrument. British Journal of Medical Psychology, 52, 1-10, 1979.

RESNICK, H.S., ACIERNO, R., KILPATRICK, D.G. Health impact of interpersonal violence 2: Medical and mental health outcomes. Behavioral Medicine, 23, 65-78, 1997.

ROBINS, L. The natural history of drug abuse. In: Lettleri D, Sayers M and Pearson H (eds) Evaluation of treatment of drug abuser. Acta Psychiatrica Scandinavica (suppl 184), 7-20, 1980.

ROOSA, M., SANDER, I., BEALS, J., SHORT, J. Risk status of adolescent children of problem-drinking parents. American Journal of Community Psychology, 16, 225-446, 1988.

ROUNSAVILLE, B.J., WEISSMAN, M.M., WILBERM C.H., KLEBER, H.D. Pathways of opiate addiction: an evaluation of differing antecedents. The British Journal of Psychiatry, 141, 437-446, 1982.

SARVELA, P., FORD, T. Indicators of substance use among pregnant adolescents in the Mississippi Delta. Journal of school Health, 41, 47-48, 1992.

SIMS, A., SIMS, D. The phenomenology of post-traumatic stress disorder. A symptomatic study of 70 victims of psychological trauma. Psychopathology, 31, 96-112, 1998.

SMITH, G., SCHWERIN, F., STUBBLEFIELD. Licit and illicit substance use by adolescents: Psychosocial predisposition and escalatory outcome. Contemporary Drug Problem, 75-100, 1982. 
STIMSON, G.V. Heroin and behavioural diversity among addicts attending London clinics, Irish University Press, Shannon, 1973.

STOKER, A., SWADI, H. Perceived family relationships in drug abusing adolescents. Drug and Alcohol Dependence, 25, 293-297, 1990.

SWADI, H. Individual risk factors for adolescent substance use. Drug and Alcohol Dependence, 55, 209-224, 1999.

TENNANT, C., BERNARDI, E. Childhood loss in alcoholics and narcotic addicts. British Journal of Addiction, 83, 695-703, 1988.

TODD, G.F. Reliability of statements about smoking habits, Tobacco Research Council, Research Paper 2A (London, Tobacco Research Council), 1966.

TROOP, N.A., TREASURE, J.L. Psychosocial factors in the onset of eating disorders: response to life events and difficulties. British Journal of Medical Psychology, 70, 373385, 1997.
WILLS, T., VACCARO, D., McNAMARA, G. Novelty seeking, risk taking, and related constructs as predictors of adolescent substance use: an application of Cloninger's Theory. Journal of Substance Abuse, 6, 1-20, 1994.

WILLS, T, DU HAMEL, K., VACCARO, D. Activity and mood temperament as predictors of adolescent substance use: test of a self-regulation mediational model. Journal of Personality and Social Psychology, 68, 901-916, 1995.

Dirección para correspondencia:

Hamdy Fouad A Moselhy

consultant Psychiatrist

Sandwell Mental Health NHS Trust

Anchor Project

2 St Michael s Court

Victoria Street

West Bromwich

West Midlands

B70 8ET

UNITED KINGDOM 